\title{
SUBGROUPS WITH CENTRE IN HNN GROUPS
}

\author{
A. KARRASS and D. SOLITAR* \\ (Received 4 February 1975; revised 17 September 1976) \\ Communicated by M. F. Newman
}

\begin{abstract}
A subgroup with non-trivial centre in a one-relator group is shown to be a treed HNN group (graph product) with infinite cyclic vertices. Moreover, subgroups with non-trivial centre in HNN groups are also examined.
\end{abstract}

\section{Introduction}

Karrass, Pietrowski and Solitar (1974) have proved the following:

If $G$ is a one-relator group and $H$ is a finitely generated subgroup with non-trivial centre, then $H$ is a treed $H N N$ group with infinite cyclic vertices. (A treed HNN group is an HNN group whose base is a tree product and whose associated subgroups are contained in vertices of the tree product base).

Here we establish the result for $H$ infinitely generated. More generally, if $G$ is a group built up from the infinite cyclic group by repeatedly forming free products and HNN extensions always using free amalgamated and associated subgroups, and $H$ is a subgroup with nontrivial centre, then $H$ is a treed $H N N$ group with infinite cyclic vertices.

As a corollary we obtain that $H$ is an extension of a free group by a subgroup of the additive rationals; this was first proved by Cossey and Smythe (1975). As another corollary we have that if $H_{1}<H$ and $H_{1}$ has trivial intersection with the centre of $H$, then $H_{1}$ is a free group; this result was first proved for $H_{1}$ finitely generated in Karrass, Pietrowski and Solitar (1974) and extended to infinitely generated $H_{1}$ in Bieri and independently in Bagherzadeh (1976).

Moreover, let $G$ be a generalized free product

* This Research was supported in part by Canada Council Leave Fellowships. 


$$
G=(A * B ; U)
$$

or an HNN group

$$
G=\left\langle t, K ; t L t^{-1}=M\right\rangle
$$

and let $H$ be a subgroup of $G$ with $g$ a central element of $H$ not contained in a conjugate of $U$ or of $L$. If $G$ is as in (1), then $H$ is in a conjugate of $A$ or $B$, or $H$ is an infinite cyclic extension of a subgroup of a conjugate of $U$. Similarly, if $G$ is as in (2), then $H$ is in a conjugate of $K$, or $H$ is an infinite cyclic extension of a subgroup of a conjugate of $L$.

We wish to thank the referee for his helpful comments.

\section{Parity and sign in HNN groups}

Let $G$ be as in (2) and let $L_{\varepsilon}$ denote $L$ when $\varepsilon=1$ and $M$ when $\varepsilon=-1$. A reduced form in $G$ is a product

$$
k_{0} t^{i} k_{1} t^{2} \cdots t^{n} k_{n}
$$

where $\varepsilon_{i}= \pm 1, k_{i} \in K$, and if $\varepsilon_{i+1}=-\varepsilon_{i}$ then $k_{i} \notin L_{\varepsilon_{i}} ; n$ is called the $t$-length of (3). [Britton's lemma implies that a reduced form (3) defines an element $\neq 1$ if either $n>0$ or $k_{0} \neq 1$.] A block $B$ is a product (3) where $n>0, k_{0}=$ $k_{n}=1, \varepsilon_{i}=\varepsilon$ for $i=1, \cdots, n ; \varepsilon$ is the sign of $B$. Clearly, a reduced form (3) can be written in block form

$$
k_{0} B_{1} k_{i_{1}} B_{2} k_{i_{2}} \cdots B_{r} k_{i r}
$$

where the $B_{j}$ alternate in sign and $k_{i}, \neq 1$ for $j \neq r ; r$ is the block-length of (3).

Suppose right coset representative systems for $K \bmod L$ and $K \bmod M$, respectively, are chosen. Then a reduced form (3) is called normal if $k_{i}$ is a representative for $K \bmod L_{i}, i>0$; every element in $G$ has a unique normal form (this follows easily from Britton's lemma).

LEMMA 1. Let the reduced form

$$
p_{0} t^{\delta_{1}} p_{1} t^{\delta_{2}} \cdots t^{\delta_{m}} p_{m}
$$

define the same element as the reduced form (3). Then (3) and (4) have the same $t$-length (i.e., $m=n$ ), and $\varepsilon_{i}=\delta_{i}$; moreover, $k_{i}$ and $p_{i}$ determine the same double coset of $K \bmod \left(L_{r_{t}}, L_{-r_{1+1}}\right)$ where $L_{r_{0}}=L_{-\varepsilon_{n+1}}=1$. In particular, any two reduced forms of an element of an HNN group have the same block length and corresponding blocks have the same t-length and sign.

Proof. It is easily shown by induction on the $t$-length of (3), that if (4) is the normal form for (3), then the conclusions of the lemma are satisfied. 
Specifically, (3) is normalized by first normalizing $k_{1} t^{\varepsilon_{2}} k_{2} \cdots t^{\varepsilon_{n}} k_{n}$ to $p_{1}^{\prime} t^{\varepsilon_{2}} p_{2} \cdots t^{\varepsilon_{n}} p_{n} \quad$ and then $k_{0} t^{\varepsilon_{1}} p_{1}^{\prime} t^{\varepsilon_{2}} p_{2} \cdots t^{\varepsilon_{n}} p_{n}$ is normalized to $p_{0} t^{f_{1}} p_{1} t^{f_{2}} p_{2} \cdots t^{\varepsilon_{n}} p_{n}$.

If $g \in G$, then $\tau(g), \beta(g)$ denote the $t$-length and block-length of $g$. The sign of $g$ is the sign of the first block of $g$; the cosign of $g$ is the sign of the last block of $g$. An element of $G$ not in $K$ is odd or even according as it has odd or even block length; an element of $G$ not in $K$ is positive or negative according as its sign is positive or negative.

LEMMA 2. Let $G$ be as in (2). Then an odd element cannot commute with an even element, and a positive even element cannot commute with a negative even element.

Proof. It is easy to see that if $v, w$ are in $G-K$, and $\operatorname{cosign} v=\operatorname{sign} w$, then $\tau(v w)=\tau(v)+\tau(w), \beta(v w)=\beta(v)+\beta(w)-1, \operatorname{sign} v w=\operatorname{sign} v$, and cosign $v w=\operatorname{cosign} w$. On the other hand, if cosign $v \neq \operatorname{sign} w$, then $\tau(v w)<$ $\tau(v)+\tau(w)$ or $\beta(v w)=\beta(v)+\beta(w)$.

Suppose $v w=w v$. Then $\operatorname{cosign} v=\operatorname{sign} w$ iff $\operatorname{cosign} w=\operatorname{sign} v$.

Hence, if $v$ is odd, $\operatorname{sign} v=\operatorname{cosign} v$ and so sign $w=\operatorname{cosign} w$; hence $w$ is odd. Also if $v$ is positive even, then $w$ is even; if $w$ were negative, then sign $v=\operatorname{sign} v w=\operatorname{sign} w v=\operatorname{sign} w$, which implies $w$ is positive.

Corollary 1. Let $G$ be as in (2) with $K \neq L$. Then the centre of $G$ is in $L$.

Proof. Since $K \neq L, G$ contains an even element $t k t^{-1}, k \notin L$, as well as the odd element $t$. Therefore, the centre of $G$ is in $K$ and hence in $t^{-1} K t$. Since $L=K \cap t^{-1} K t$, the centre of $G$ is in $L$.

The following corollary, although not needed for the proofs of Theorems 1 or 2 , is also a consequence of Lemma 2 . It was obtained independently in Bagherzadeh (1976) and by L. Comerford (unpublished).

Corollary 2. Let $G$ be as in (2) with $L \neq K$ or $M \neq K$. If $G$ is a non-trivial direct product $C \times D$, then $C$ or $D$ is in $L$.

Proof. Since $t=c d$ where $c \in C$ and $d \in D, c$ and $d$ commute with $t$; hence $c$ or $d$ is odd. To be specific suppose $d$ is odd. Then by Lemma $2, C$ cannot contain even elements. We show, however, that $C$ does contain even elements if $C \nless L$.

Clearly, $C \nless K$ since $K \cap t^{-1} K t=L$. Assume $K \neq L_{\varepsilon}$. If $C$ contains an element $k_{0} B_{1}$ of block length one and sign $\varepsilon$, then $C$ contains $k_{0} B_{1} k B_{1}^{-1} k_{0}^{-1} k^{-1}$ where $k \notin L_{\epsilon}$; hence if $L \neq K(M \neq K), C$ would contain a positive even (negative even) element. If $g \in C$ with $g=k_{0} B_{1} \cdots B_{n}, n \geqq 2, n$ odd, sign $B_{n}=\varepsilon$, then $g$ is cyclically reduced of block length $\geqq 2$; but cyclic 
permutations of a cyclically reduced element of block length $\geqq 2$, will yield positive and negative even elements.

COROLLARY 3. If a one-relator group $G$ is a proper direct product, then $G$ is finite cyclic or free abelian of rank two.

Proof. Let $G=C \times D, C \neq 1 \neq D$, and suppose $G$ is not cyclic. We may Nielsen transform the generators of $G$ so that the corresponding relator is in pre-abelianized form (see p. 142 in Magnus, Karrass, and Solitar (1966)) and will have zero exponent sum on one of the generators, say $t$; moreover, since $G$ cannot be a proper free product, when rewritten in terms of the conjugates of the other generators by $t$, this relator will involve distinct conjugates of at least one generator. Then by the standard method for writing a one-relator group as an HNN extension of another one-relator group (see Moldavanski (1967)), $G=\left\langle t, K ; t L t^{-1}=M\right\rangle$ where $L$ is free, $K=g p(L, M)$, and $K$ is a one-relator group on rank $L+\operatorname{rank} G-1$ generators (provided we include in $K$ at least two consecutive conjugates of every generator $\neq t$ of $G$ ).

If $K \neq L$ or $K \neq M$, then by the preceding corollary, we may suppose $C<L$. Since $L$ is free, $D \cap L=1$ and so $C=L$. But $C \triangleleft G$, so that $M=L=K$.

Thus $L=K=M$ and hence rank $G \leqq \operatorname{rank} K+1-\operatorname{rank} L+1=2$. On the other hand, $C$ and $D$ must be free (Corollary 2 to Theorem 2 in Karrass, Pietrowski and Solitar (1974)), and homomorphic images of $G$; but if $C$ or $D$ had rank 2 than $G=C \times D$ would have rank $\geqq 3$. Consequently $C$ and $D$ must be cyclic and $G$ free abelian of rank two.

LEMMA 3. Let $G$ be as in (2), $H<G$, and suppose $H$ contains an odd element. Then $H$ contains a positive even element unless $H$ is conjugate (by an element of $K)$ to an $H N N$ group $\left\langle s, L^{\prime} ; s L^{\prime} s^{-1}=L^{\prime \prime}\right\rangle$ where $L^{\prime \prime}<L^{\prime}<L$; if $H-K$ contains only odd elements, then $L^{\prime}=L^{\prime \prime}$.

Proof. By induction on $\tau(u)$, one can easily show that if $u, v$ are in $G-K$ and $\tau(u) \geqq \tau(v)$, then one of the following three possibilities holds: $\operatorname{sign} u v=\operatorname{sign} u$ and cosign $u v=\operatorname{cosign} v$; or $\tau(u v)<\tau(u)$ and sign $u v=\operatorname{sign} u$; or $u v \in K$.

Now let $s$ be a positive odd element of shortest $t$-length in $H$, and suppose $H$ contains no positive even elements. We show that $K \cap H=$ $k L^{\prime} k^{-1}$ where $L^{\prime}<L, k \in K$, and that $H=g p(s, K \cap H)$. If $s$ has reduced form as in (3), and $k^{\prime} \in K \cap H$, then $s k^{\prime} s^{-1}$ will be positive even unless $k_{n} k^{\prime} k_{n}^{-1} \in L$; hence $K \cap H=k_{n}^{-1} L^{\prime} k_{n}$ where $L^{\prime}<L$. Moreover, if $u$ is any positive element in $H$, then $u$ is odd and $\tau(u) \geqq \tau(s)$. Since $u s^{-1}$ is not positive even, our first remark implies that $u s^{-1} \in K$, or $\tau\left(u s^{-1}\right)<\tau(u)$ and $u s^{-1}$ is positive. Hence by induction on the $t$-length of $u$, every positive element of $H$ 
has the form $p s^{n}, n>0, p \in K \cap H$; moreover $\tau\left(p s^{n}\right)=n \tau(s)$. Therefore $s(K \cap H)$, which consists of positive elements having the same $t$-length as $s$, is contained in $(K \cap H) s$, so that $s(K \cap H) s^{-1}=K^{\prime}<K \cap H$. Hence the HNN group $R=\left\langle s, K \cap H ; s(K \cap H) s^{-1}=K^{\prime}\right\rangle$ can be mapped homomorphically onto $H_{1}=g p(s, K \cap H)$ in $H$; thus the normal subgroup generated by $K \cap H$ in $H_{1}$ is just the ascending union $s^{-m}(K \cap H) s^{m}, m \geqq 0$, and so has trivial intersection with $g p(s)$ in $H_{1}$ since $g p(s) \cap(K \cap H)=1$; thus the homomorphism of $R$ onto $H_{1}$ has kernel in the normal closure of $K \cap H$ in $R$. But this is mapped isomorphically onto the normal closure of $K \cap H$ in $H_{1}$; hence, $R$ is mapped isomorphically onto $H_{1}$.

To show that $H_{1}=H$, it remains to consider the negative elements of $H$. Clearly, any negative odd element of $H$ is in $H_{1}$ since it has the form $s^{-n} p^{-1}, n>0, p \in K \cap H$. Let $q$ be a negative even element of smallest $t$-length in $H-H_{1}$. If $\tau(q) \leqq \tau(s)$, then by our initial remark, $s q \in H_{1}$ contrary to $q \notin H_{1}$; if $\tau(q)>\tau(s)$ then $q s^{-1} \in H_{1}$, or $\tau\left(q s^{-1}\right)<\tau(q)$ and $q s^{-1}$ is negative even, contrary to the definition of $q$.

Finally, if $H$ contains no negative even element, then $s^{-1}(K \cap H) s<$ $K \cap H$, since $\left(s^{-1} k\right) s$ cannot be negative and of smaller $t$-length than $s$.

An analogous result clearly holds for subgroups $H$ of $G$ which contain no negative even elements.

Using similar reasoning we obtain analogous results for an amalgamated product (1). Specifically, call the elements of $A-U$ positive and of $B-U$ negative; a non-trivial product $g_{1} g_{2} \cdots g_{n}$ with factors $g_{i}$ alternating from $A-U$ and $B-U$ has the sign of $g_{1}$ and the parity of $n$.

LEMMA 4. Let $G$ be as in (1). Then an even element cannot commute with an odd element, and a positive odd element cannot commute with a negative odd element.

LemMA 5. Let $G$ be as in (1), $H<G$, and suppose $H$ contains an even element. Then $H$ contains both a positive odd element and a negative odd element unless $H$ is conjugate to an $H N N$ group $\left\langle s, U^{\prime} ; s U^{\prime} s^{-1}=U^{\prime \prime}\right\rangle$ where $U^{\prime \prime}<U^{\prime}$ and $U^{\prime}=H \cap U$; if $H-U$ contains only even elements then $U^{\prime}=U^{\prime \prime}$.

\section{Subgroups with centre}

TheORem 1. (i) Suppose $G$ is as in (1), $H<G, g$ is in the centre of $H$, and $g$ has no conjugate in $U$. Then $H$ is conjugate to an $H N N$ group $\left\langle s, U^{\prime} ; s U^{\prime} s^{-1}=U^{\prime}\right\rangle$ where $U^{\prime}<U$ (i.e., $H$ is an infinite cyclic extension of a subgroup of a conjugate of $U$ ) unless $H$ is in a conjugate of a factor.

(ii) Suppose $G$ is as in (2), $H<G, g$ is in the centre of $H$, and $g$ has no 
conjugate in $L$. Then $H$ is conjugate to an $H N N$ group $\left(s, L^{\prime} ; s L^{\prime} s^{-1}=L^{\prime}\right\rangle$ where $L^{\prime}<L$ unless $H$ is in a conjugate of $K$.

PROOF. (i) If $g$ is in a factor then $C(g)$, the centralizer of $g$, is also in a factor. If no conjugate of $g$ is in a factor, then we may assume $g$ is cyclically reduced and even. Hence by Lemma $4, H$ has no odd elements and so $H$ has the asserted structure by Lemma 5 .

(ii) If $g \in K$ then again an easy argument shows that $C(g)<K$. Suppose no conjugate of $g$ is in $K$. If some conjugate $H^{x}$ of $H$ contains an odd element, then by Lemma $2, g^{x}$ is odd and so $H$ has the asserted structure by Lemmas 2 and 3.

Suppose no conjugate of $H$ contains an odd element. Now some cyclic rearrangement of a cyclically reduced element of $G$ is odd unless all of its blocks have $t$-length 1 . Hence an element of $G$ having no odd conjugate must be in a conjugate of $Q=\left\langle K * t K t^{-1} ; M=t L t^{-1}\right\rangle$. Thus $H<K^{G}$. We may assume $g \in Q$, and show $H<Q$. Now $K^{G}$ is a tree product of three groups $Q_{-1}, Q, Q_{1}$, where $Q_{-1}$ is generated by all $t^{-n} K t^{n}$ for $n \geqq 1, Q_{1}$ is generated by all $t^{m} K^{-m}$ for $m \geqq 2$, with $t^{-1} M t=L$ amalgamated between $Q_{-1}$ and $Q$, and $t M t^{-1}=t^{2} L t^{-2}$ amalgamated between $Q$ and $Q_{1}$. Since $g$ is not in a conjugate of $L$, the amalgamations are proper and so by the first remark in the proof of (i) above, $H<Q$. Since $g$ is not in a conjugate of $K$, (i) applies again to show that $H$ has the asserted form.

COROLLARY. If $g$ is an odd element in (2), then $C(g)$, the centralizer of $g$, is an infinite cyclic extension of a subgroup of a conjugate of $L$.

\section{One relator groups and generalizations}

We next wish to describe the structure of subgroups with non-trivial centre of one-relator groups. As is well known (and follows from Lemma 4) the centre of an amalgamated product $G=(A * B ; U)$ is in $U$ if $A \neq U \neq B$. Similarly, the centre of a tree product is in each edge if the tree product is proper, i.e., if it has at least two vertices and for each edge $U_{i j}$ joining $A_{i}$ to $A_{i}, A_{i} \neq U_{i j} \neq A_{j}$. On the other hand, if the tree product is not proper, its centre need not be contained in any edge, e.g., consider a properly ascending union of groups. However, this is essentially the only exception.

Lemma 6. Let $G$ be given as a tree product $P$. If $G$ is not an ascending union of some of its vertices, then $G$ can be expressed as a tree product $P^{\prime \prime}$ of a subset of the original vertex groups and edge groups of $P$ in such a way that the centre of $G$ is in each edge group; moreover, each of the vertices of $P$ is contained in a vertex group of $P^{\prime \prime}$.

Proof. We wish to write $P$ as a proper tree product. Let $A_{0}$ be a vertex 
group of $P$ and define the level $\lambda\left(A_{i}\right)$ of the vertex group $A_{i}$ as the length of the shortest path in $P$ from $A_{0}$ to $A_{i}$. Clearly, every subtree of $P$ has a unique vertex of minimal level.

An edge of $P$ joining $A_{i}$ to $A_{j}$ with $\lambda\left(A_{i}\right)<\lambda\left(A_{i}\right)$ is said to be descending (strictly ascending) if $A_{j}$ is contained in $A_{i}\left(A_{j}\right.$ strictly contains $A_{i}$ ); a subgraph is descending (strictly ascending) if each edge of it is descending (strictly ascending). If we delete all edges of $P$ but the descending ones (strictly ascending ones), then the resulting connected components are maximum descending (strictly ascending) subtrees. Clearly the tree product of every maximum descending subtree is just its vertex of minimal level.

We shall now eliminate the descending edges from $P$ by contracting the maximum descending subtrees. By the generalized associative law for tree products (Theorem 1 of Karrass and Solitar (1970)), $G$ is the tree product $\boldsymbol{P}^{\prime}$ of each of the vertices of $P$ of minimal level in some maximum descending subtree of $P$; moreover the edges of $P^{\prime}$ are the edges of $P$ which join vertices from different maximum descending subtrees of $P$. Let $\lambda^{\prime}$ be the level function for the tree $P^{\prime}$ with $\lambda^{\prime}\left(A_{0}\right)=0$. Then $P^{\prime}$ has no descending edges. For, suppose $A_{i}, A_{j}$ are joined by an edge in $P^{\prime}$ and $A_{i}<A_{i}$. Then in $P$ there are neighboring vertices $A_{i}^{\prime}$ and $A_{j}^{\prime}$ such that there exists (in $P$ ) a descending path from $A_{i}$ to $A_{i}^{\prime}$ and from $A_{i}$ to $A_{i}^{\prime}$. Now in $P$, the simple path joining $A_{i}$ to $A_{j}$ generates its tree product, and so $A_{i}$ is contained in each of the vertices of this simple path. Thus $A_{i}^{\prime}<A_{i}<A_{j}^{\prime}$, and hence $\lambda\left(A_{i}^{\prime}\right)<\lambda\left(A_{j}^{\prime}\right)$ since the edge joining $A_{i}^{\prime}$ and $A_{j}^{\prime}$ cannot be descending. But since $A_{j}^{\prime}$ can be joined by an edge to a unique vertex of smaller level, the vertex $A_{;}^{\prime}$ must coincide with the vertex $A_{j}$. Therefore in $P$ the simple path from $A_{0}$ to $A_{j}$ consists of the simple path from $A_{0}$ to $A_{i}$, the descending path from $A_{i}$ to $A_{i}^{\prime}$, and the edge from $A_{i}^{\prime}$ to $A_{j}$; moreover, $A_{i}\left(A_{j}\right)$ is the only vertex on this simple path joining $A_{0}$ to $A_{i}\left(A_{i}\right)$, which lies in the maximum descending subtree containing $A_{i}\left(A_{j}\right)$. When this path is projected into $P^{\prime}$ and made simple, there results a simple path in $P^{\prime}$ joining $A_{0}$ to $A_{j}$ and passing through $A_{i}$; thus $\lambda^{\prime}\left(A_{j}\right)>$ $\lambda^{\prime}\left(A_{i}\right)$. Consequently, $P^{\prime}$ has no descending edges.

We next contract slightly the maximum strictly ascending subtrees. Clearly $P^{\prime}$ is the tree product of its maximum strictly ascending subtrees, with edges the proper edges of $P^{\prime}$. Let $M$ be a maximum strictly ascending subtree of $P^{\prime}$. If $M$ is a chain and some proper edge of $P^{\prime}$ has a vertex in $M$, let $A_{i}$ be the vertex of $M$ of minimum level on a proper edge; let $M^{\prime}$ result from $M$ by contracting the vertices below $A_{i}$ into $A_{i}$. If $M$ is not a chain, then there exists a unique vertex $\boldsymbol{A}_{i}$ of minimum level which is joined in $M$ to at least two vertices of higher level; let $M^{\prime}$ result from $M$ by contracting the vertices below $A_{i}$ into $A_{i}$. Let $P^{\prime \prime}$ result from $P^{\prime}$ by replacing each $M$ by $M^{\prime}$. 
Suppose now that $G$ is not an ascending union of vertices. Then $Z$, the centre of $G$, will be contained in each edge of $P^{\prime \prime}$. For, $Z$ is contained in each proper edge of $P^{\prime \prime}$ and so is in each maximum strictly ascending subtree $M^{\prime}$ of $P^{\prime \prime}$. If $M^{\prime}$ is a chain then, by construction, there is a proper edge adjacent to the lowest vertex of $M^{\prime}$ and so $Z$ is contained in each vertex and edge of $M^{\prime}$. If $M^{\prime}$ is not a chain then $M^{\prime}=(C * D ; U)$ where $U$ is the vertex of minimum level in $M^{\prime}$ and $C, D$ each contain a vertex of $M^{\prime}$ joined to $U$. Since $M^{\prime}$ is strictly ascending, $C \neq U \neq D$; hence $Z<U$. But each vertex of $M^{\prime}$ contains $U$ and so each edge of $M^{\prime}$ contains $Z$.

Corollary. Let $G$ be given as a tree product $P$. If $G$ is not an ascending union of vertices of $P$, then $G$ can be expressed as a proper tree product $Q$ whose edge groups are edge groups from $P$ and whose vertices are strictly ascending trees with vertex groups and edge groups from $P$.

LEMMA 7. Let $G$ be a treed HNN group with tree product base $B$ having vertices $\left\{A_{i}\right\}$ where each $A_{i}$ is itself a treed HNN group such that each amalgamated and associated subgroup of $G$ incident with $A_{i}$ is conjugate in $A_{i}$ to a subgroup of a vertex of $A_{i}$. Then $G$ is a treed $H N N$ group whose base $C$ is a tree product with vertices conjugate in $B$ to the vertices of the various $A_{i}$ and whose amalgamated subgroups are conjugate in $B$ to the original amalgamated subgroups of $G$ and to the amalgamated subgroups of the various $A_{i}$; finally, the new associated subgroups are conjugate in $B$ to the original associated subgroups of $G$ and to the associated subgroups of the various $A_{i}$.

Proof. Let $A_{0}$ be a vertex of $B$ and let $\lambda$ be the level function for $B$ with $\lambda\left(A_{0}\right)=0$. For each vertex $A_{1}$ in $B$ of level one, there exists an edge $U_{01}=U_{10}$ joining $A_{01}$ to $A_{1}$. By hypothesis $a_{01} U_{01} a_{01}^{-1}$ and $a_{10} U_{10} a_{10}^{-1}$ are in vertices of $A_{0}$ and $A_{1}$ respectively, where $a_{i j} \in A_{1}$. If we define $A_{i}=$ $a_{01} a_{11}^{-1} A_{1} a_{10} a_{01}^{-1}, \quad$ then $\quad g p\left(A_{0}, A_{1}\right)=g p\left(A_{0}, A_{1}^{\prime}\right)=\left(A_{0} * A_{1}^{\prime} ; a_{01} U_{01} a_{01}^{-1}\right.$ $\left.=a_{01} U_{11} a_{01}^{-1}\right)$, and the amalgamated subgroups are in vertices of the treed HNN groups $A_{1}$ and $A_{1}^{\prime}$. Similarly, each vertex $A_{2}$ of level two can be joined by an edge $U_{21}=U_{12}$ to a vertex $A_{1}$ of level one, where $a_{12} U_{12} a_{12}^{-1}$ and $a_{21} U_{21} a_{21}^{\prime}$ are in vertices of $A_{1}$ and $A_{2}$, respectively. If we define $A_{2}^{\prime}=$ $a_{01} a_{10}^{-1} a_{12} a_{21}^{-1} A_{2} a_{21} a_{12}^{-1} a_{11} a_{11}^{1}$, then

$$
\begin{aligned}
g p\left(A_{0}, A_{1}, A_{2}\right) & =g p\left(A_{01}, A_{1}^{\prime}, A_{2}^{\prime}\right)=\left\langle A_{0} * A_{1}^{\prime} * A_{2}^{\prime} ; a_{01} U_{01} a_{01}^{-1}=\right. \\
& \left.=a_{01} U_{10} a_{01}^{-1}, a_{01} a_{10}^{-1} a_{12} U_{12} a_{12}^{-1} a_{10} a_{01}^{\prime \prime}=a_{01} a_{10}^{-1} a_{12} U_{21} a_{12}^{-1} a_{10} a_{01}^{-1}\right\rangle
\end{aligned}
$$

where the amalgamated subgroups are in vertices of the treed HNN groups $A_{0}, A_{1}^{\prime}, A_{2}^{\prime}$. Continuing in this way we obtain that $B$ is a treed HNN group whose tree product base $C$ has as vertices conjugates in $B$ of the vertices of 
the various $A_{i}$; moreover, the amalgamated subgroups of $C$ are conjugate in $B$ to the original amalgamated subgroups of $B$ and the amalgamated subgroups of the various $A_{i}$. The associated subgroups of $B$ are conjugate in $B$ to the associated subgroups of the various $A_{i}$.

Finally, consider any pair $L, M$ of associated subgroups of $G$ and the corresponding free generator $t$ of $G$. By hypothesis, $L, M$ are conjugate in $B$ to subgroups of vertices of $C$. If $b_{1} L b_{1}^{-1}$ and $b_{2} M b_{2}^{-1}$ are in vertices of $C$ where $b_{1}, b_{2} \in B$, replace $t$ by $t_{1}=b_{2} t b_{1}^{-1}$; the pair of associated subgroups corresponding to $t_{1}$ are $b_{1} L b_{1}^{-1}$ and $b_{2} M b_{2}^{-1}$. In this way $G$ can be expressed as a treed HNN group of the type asserted in Lemma 7.

Lemma 7 also appears in Cohen (1973).

LemmA 8. Suppose $G / N$ is a treed $H N N$ group with vertices $\boldsymbol{A}_{i}$, amalgamated subgroups $U_{i j}=U_{i i}$, and associated subgroups $L_{k}, M_{k}$ corresponding to the generator $t_{k}$. If $\varphi$ is the natural homomorphism of $G$ onto $G / N$, then $G$ is a treed HNN group with vertices $\varphi^{-1}\left(A_{i}\right)$, amalgamated subgroups $\varphi^{-1}\left(U_{i j}\right)=$ $\varphi^{-1}\left(U_{j i}\right)$, and associated subgroups $\varphi^{-1}\left(L_{k}\right), \varphi^{-1}\left(M_{k}\right)$ corresponding to the generator $t_{k}^{\prime}$ where $t_{k}^{\prime}$ is an element of $\varphi^{-1}\left(t_{k}\right)$.

Proof. First we observe that the group $G=(A * B ; U)$ can be characterized internally by the fact that $G=g p(A, B), A \cap B=U$, and $g_{1} g_{2} \cdots g_{n} \neq 1$ where the $g_{i}$ are alternately from $A-U$ and $B-U$; the group $G=\left\langle t, K ; t L_{1} t^{-1}=L_{-1}\right\rangle$ can be characterized internally by the fact that $G=g_{P}(t, K), t L_{1} t^{-1}=L_{-1}$, and $k_{0} t^{\varepsilon_{2}} k_{1} t^{\varepsilon_{2}} k_{2} \cdots t^{\varepsilon_{n}} k_{n} \neq 1$, where $k_{i} \in K, \varepsilon_{i}=$ \pm 1 , and $k_{i} \notin L_{r}$ if $\varepsilon_{i}=\varepsilon$ and $\varepsilon_{i+1}=-\varepsilon$.

If $G / N=(A * B ; U=V)$ or $\left\langle t, K ; t L t^{-1}=M\right\rangle$, then the result follows from the internal characterizations of such groups. If $G / N$ is a treed HNN group with finitely many vertices and free part of finite rank, then the result follows by induction. Finally, one can characterize a general treed HNN group internally by specifying that each finite subtree of vertices and a finite number of free generators whose associated subgroups are incident with these vertices generate the natural treed HNN group.

THEOREM 2. Let $H$ be a subgroup of a torsion-free one relator group $G$ where $H$ has non-trivial centre $Z$. Then $H$ is a treed $H N N$ group with infinite cyclic vertices; moreover, if $H$ is not locally cyclic and not free abelian of rank two, then $Z$ is contained in each vertex and associated subgroup of this treed HNN group.

Proof. The proof is by induction on the length of the relator of $G$. If $G$ is not infinite cyclic, then $G$ is a subgroup of an $H N N$ group $\left\langle t, K ; t L t^{-1}=M\right\rangle$ where $K$ is a group with a single defining relator of shorter length than that of 
$G$, and $L$ is free. Therefore $H$ is a treed HNN group with vertices in conjugates of $K$ and amalgamated and associated subgroups in conjugates of $L$ (by Theorem 1 of Karrass, Pietrowski and Solitar (1974)).

Suppose first that $H$ has non-trivial free part and $Z$ is contained in each associated subgroup of $H$. Then $Z$ must be infinite cyclic and contained in some vertex of the tree product base $B$ of $H$. Hence by Lemma 6 we may assume that $Z$ is contained in each vertex and amalgamated subgroup of $B$. By inductive hypothesis each vertex $V$ of $H$ is a treed $H N N$ group with infinite cyclic vertices $V_{\text {i }}$ each of which contains $Z$ (this holds even if $V$ is locally cyclic or free abelian of rank two). If $E$ is any amalgamated or associated subgroup incident with $V$ in the treed HNN representation of $H$, then $E$ is infinite cyclic since it is free and has a non-trivial centre and $E / Z$ is a finite subgroup of the treed $H N N$ group $V / Z$, which has vertices $V_{i} / Z$. Hence a conjugate of $E / Z$ in $V / Z$ is in some vertex $V_{i} / Z$. Therefore some conjugate of $E$ in $V$ lies in some vertex $V_{i}$. It follows from Lemma 7 that $H$ is a treed HNN group with infinite cyclic vertices such that $Z$ is contained in each vertex and amalgamated and associated subgroup.

If the free part $F$ of $H$ has rank $\geqq 2$, or if $F$ is infinite cyclic and the base of $H$ is different from either associated subgroup, then by Corollary 1 to Lemma 2, the preceding argument applies.

Hence if $F \neq 1$ we may assume $H$ is an infinite cyclic extension of a free group $B$; moreover, $H / Z$ is a finite extension of the free group $B Z / Z$. Therefore by Scott (1974), $H / Z$ is a treed HNN group with finite vertices, and so by Lemma $8, H$ is a treed $H N N$ group with infinite cyclic vertices and $Z$ is contained in each vertex and associated subgroup.

Finally, suppose $F=1$. If $H=B$ is not a single vertex, then Lemma 6 shows that $H$ is locally cyclic or that $Z$ is contained in each vertex $V$ of $B$, and so as in the first case $H$ has the desired form. If $H=B$ is a single vertex, then the inductive hypothesis applies.

Corollary 1. Let $A, B, K$ be groups such that any subgroup $H$ with non-trivial centre has the structure described in the preceding theorem. If $G=(A * B ; U)$ or $G=\left\langle t, K ; t L t^{-1}=M\right\rangle$ where $U, L$ are free, then any subgroup of $G$ with non-trivial centre has the structure described.

Thus, for example, the centralizer of an element in $(A * B ; U)$ where $A, B$ are free, is a treed HNN group with infinite cyclic vertices.

COROLlary 2. Let $G$ be as in the preceding corollary, and let $C$ be the centralizer of an element $x \neq 1$ in $G$. If $H$ is a subgroup of $C$ such that $H \cap g p(x)=1$, then $H$ is a free group. 
ProOF. Same as for Corollary 2 of Theorem 2 in Karrass, Pietrowski and Solitar (1974).

COROllary 3. Let $G$ be as in the preceding corollary and let $H=$ $C \times D<G, C \neq 1 \neq D$. Then $C, D$ are free groups.

Proof. If $1 \neq d \in D$, then $C$ is in the centralizer of $D$, and $C \cap g p(d)=$ 1 , and so Corollary 2 applies.

In particular, if $G$ is a torsion-free one-relator group, and $C \times D<$ $G, C \neq 1 \neq D$, then $C, D$ are free.

To obtain the result of Cossey-Smythe referred to in the introduction we must establish that a treed HNN group with infinite cyclic vertices and non-trivial centre is an extension of a free group by a subgroup of the additive rationals. For this purpose choose some fixed generator $x_{n}$ of a vertex (referred to as level zero) and map this into the rational number 1 . If $x_{n+1}$ is a generator of a vertex $A_{n+1}$ of level $n+1$ and $x_{n}$ is a generator of a vertex $\boldsymbol{A}_{n}$ of level $n$ joined to $a_{n+1}$ by an edge and if the amalgamated subgroups of this edge are $x_{n}^{p}=x_{n+1}^{q}$ and $x_{n}$ has been mapped into $r_{n}$ then map $x_{n+1}$ into $p r_{n} / q$; map each free generator $t$ into 1 . If $c$ is a non-trivial central element then $c=x_{n}^{m}=x_{s}^{\prime}$ and so if $t x_{n}^{\alpha} t^{-1}=x_{s}^{\beta}$ then $t x_{n}^{\alpha m} t^{-1}=x_{s}^{\beta m}=t c^{\alpha} t^{-1}=c^{\alpha}=x_{s}^{\alpha j}$ and therefore $\beta m=\alpha j$. But if $x_{i}$ goes into the rational $r_{i}$ then $m r_{n}=j r_{s}$. Hence. $t x_{n}^{\alpha} t^{-1}$ goes into $\alpha r_{n}$ while $x_{s}^{\beta}$ goes into $\beta r_{s}$; but $\alpha / \beta=m / j=r_{s} / r_{n}$ so that $\alpha r_{n}=\beta r_{s}$ and the homomorphism is well-defined. Since the mapping is one-one on the vertices it follows that the kernel is a free group.

\section{References}

G. H. Bagherzadeh (1976). 'Commutativity in one-relator groups', J. London Math. Soc. (2) 13. $454-471$.

R. Bieri (to appear). Duality groups and groups of cohomological dimension two that are extensions'.

Daniel E. Cohen (1973). 'Groups with the free subgroups of finite index'. Conference on Group Theory. University of Wisconsin-Parkside 1972 (Lecture Notes in Mathematics. 319, 26-44. Springer-Verlag. Berlin. Heidelberg. New York, 1973)

John Cossey and N. Smythe (1975). 'HNN groups and groups with center', Knots, Groups, and 3-manifolds (Annals of Mathematics Studies. 84, 87-99. Princeton University Press and University of Tokyo Press. Princeton. New Jersey. 1975)

D. B. A. Epstein (1962). 'A result on free products with amalgamation'. J. London Math. Soc. 37, $130-132$.

A. Karrass. A. Pietrowski and D. Solitar (1974). 'An improved subgroup theorem for HNN groups with some applications'. Canad. J. Math. 26, 214-224

A. Karrass and D. Solitar (1970). 'The subgroups of a free product of two groups with an amalgamated subgroup: Trans. Amer. Math. Soc. 150, 227-255.

A. Karrass and D. Solitar (1971). 'Subgroups of HNN groups and groups with one defining relation: Canad. J. Math. 23, 627-6+3. 
Wilhelm Magnus, Abraham Karrass, Donald Solitar (1966), Combinatorial Group Theory: Presentations of groups in terms of generators and relations (Pure and Appl. Math. 13. Interscience [John Wiley \& Sons], New York, London, Sydney, 1966).

Д. И. Молдаванский [D. I. Moldavanskiī] (1967), 'О некоторых подгруппах групп с одним определяющип соотношеннем' [Certain subgroups of groups with one defining relation'], Sibirsk. Mat. Ž. 8, 1370-1384.

G. P. Scott (1974), 'An embedding theorem for groups with a free subgroup of finite index', Buil. London Math. Soc. 6, 304-306.

Department of Mathematics,

York University,

Downsview, Ontario M3J 1PS,

Canada. 